\title{
Cutaneous sinus tracts (or emerging sinus tracts) of odontogenic origin: a report of 3 cases
}

This article was published in the following Dove Press journal:

Clinical, Cosmetic and Investigational Dentistry

3 July 2010

Number of times this article has been viewed

\author{
Ronald S Brown' \\ Robert Jones ${ }^{2}$ \\ Tawana Feimster ${ }^{3}$ \\ Frances E Sam ${ }^{2}$ \\ 'Department of Oral Diagnostic \\ Services, Howard University College \\ of Dentistry, Washington, DC, USA; \\ ${ }^{2}$ Department of Oral and Maxillofacial \\ Surgery, Howard University College \\ of Dentistry, Washington, DC, USA; \\ ${ }^{3}$ Department of Endodontics, Howard \\ University College of Dentistry, \\ Washington, DC, USA
}

Correspondence: Ronald S Brown Howard University College of Dentistry, 600 W Street, NW, Room 406, Washington, DC 20059, USA

$\mathrm{Tel}+\mathrm{I} 2028060349$

Fax +I 2028060447

Email rbrown@howard.edu
Abstract: Three cases are presented in which patients presented with either cutaneous swelling or cutaneous sinus tracts of odontogenic origin. A cutaneous sinus tract of odontogenic origin is a pathway through the alveolar bone that typically begins at the apex of an infected tooth or of an infected portion of the dental alveolus and empties infected material (pus) through the skin. Where as the more common finding of an oral fistula is a pathway from the apical periodontal area of a tooth to the surface of the oral mucous membrane, permitting the discharge of suppurative material. Diagnosis, etiology and treatment are discussed with reference to patient history, clinical examinations, imaging, and treatment perspectives.

Keywords: dental abscess, fistula, cutaneous sinus tract, odotogenic infection

\section{Introduction}

A cutaneous sinus tract of odontogenic origin is a relatively uncommon condition. ${ }^{1,2}$ Cutaneous sinus tracts of odontogenic origin may present a diagnostic challenge. A cutaneous sinus tract of odontogenic origin is a pathway through the alveolar bone that typically begins at the apex of an infected tooth or of an infected portion of the dental alveolus and empties infected material (pus) through the skin. An oral fistulous tract is a much more common finding and is sometimes confused with a cutaneous sinus tract. A fistulous tract is a pathway from the apical periodontal area of a tooth to the surface of the oral mucous membrane (but not through the skin), permitting the discharge of suppurative material. These chronically draining cutaneous sinus tracts are often misdiagnosed and therefore, treated inappropriately. ${ }^{1-8}$ The differential diagnoses includes suppurative apical periodontitis, osteomyelitis, congenital fistula, salivary gland fistula, infected cyst, myositis, pustule, furuncle, foreign-body lesion, squamous cell carcinoma, granulomatous disorder, and deep mycotic infection. Successful therapy requires an accurate diagnosis. A vertical root fracture may present a diagnostic dilemma particularly for physicians, and therefore, the etiology of the skin lesion may be treated unsuccessfully for sometime before the correct diagnosis is established. Altogether too often, health-care clinicians may not appreciate the possibility of an odontogenic infection as the source of a cutaneous swelling or of a draining sinus tract, and therefore, initial and secondary treatment regimens may involve therapies directed at non-odontogenic diagnoses. ${ }^{1-8}$ 


\section{Case reports}

\section{Case I}

A 35-year-old male patient reported to the emergency room at University College of Dentistry in December of 2007, with a chief complaint of, "Swelling on the left side of face - there's a lot going on here." The medical history was positive for arthritis. The patient was taking only over-the-counter ibuprofen for his arthritis condition. The patient also reported a previous gonorrhea infection over 15 years ago, and a childhood asthma condition. The patient reported a one pack a day of cigarette smoking since the age of 16 .

Examination revealed a large palpable mass inferior to the left mandibular ramus (Figure 1A). On intra-oral examination, a large carious lesion on a broken-down left mandibular third molar was evident. A panoramic radiograph was exposed and revealed a periapical radiolucency (Figure 1B).

The diagnosis was consistent with an emerging cutaneous sinus tract secondary to a periapical abscess of left mandibular third molar. The patient was referred to oral and maxillofacial surgery. The problematic tooth was extracted without complication and the lesion healed uneventfully.

\section{Case 2}

A 72-year-old male patient reported to the emergency room at University College of Dentistry in August of 2007, with a chief complaint of "Referred for examination of chin lesion by dentist."

The medical history was essentially negative with the exception that the patient was taking hydrochlorothiazide for hypertension. Clinical examination revealed a draining extra-oral cutaneous lesion with slight swelling slightly inferior to the chin (Figure 2A). Intra-oral examination demonstrated a fractured mandibular left lateral incisor. A periapical radiograph was exposed and revealed an incomplete endodontic filling and compromised periodontal structures and a periapical radiolucency (Figure 2B). The diagnosis was a periapical abscess with a guarded prognosis.

The patient was referred to Oral and Maxillofacial Surgery for extraction. The extraction procedure was routine and the lesion healed satisfactorily with minimal scarring.

\section{Case 3}

A 53-year-old male patient reported to the emergency room at University College of Dentistry in April of 2008, with a chief complaint of "Bad teeth, need to come out."
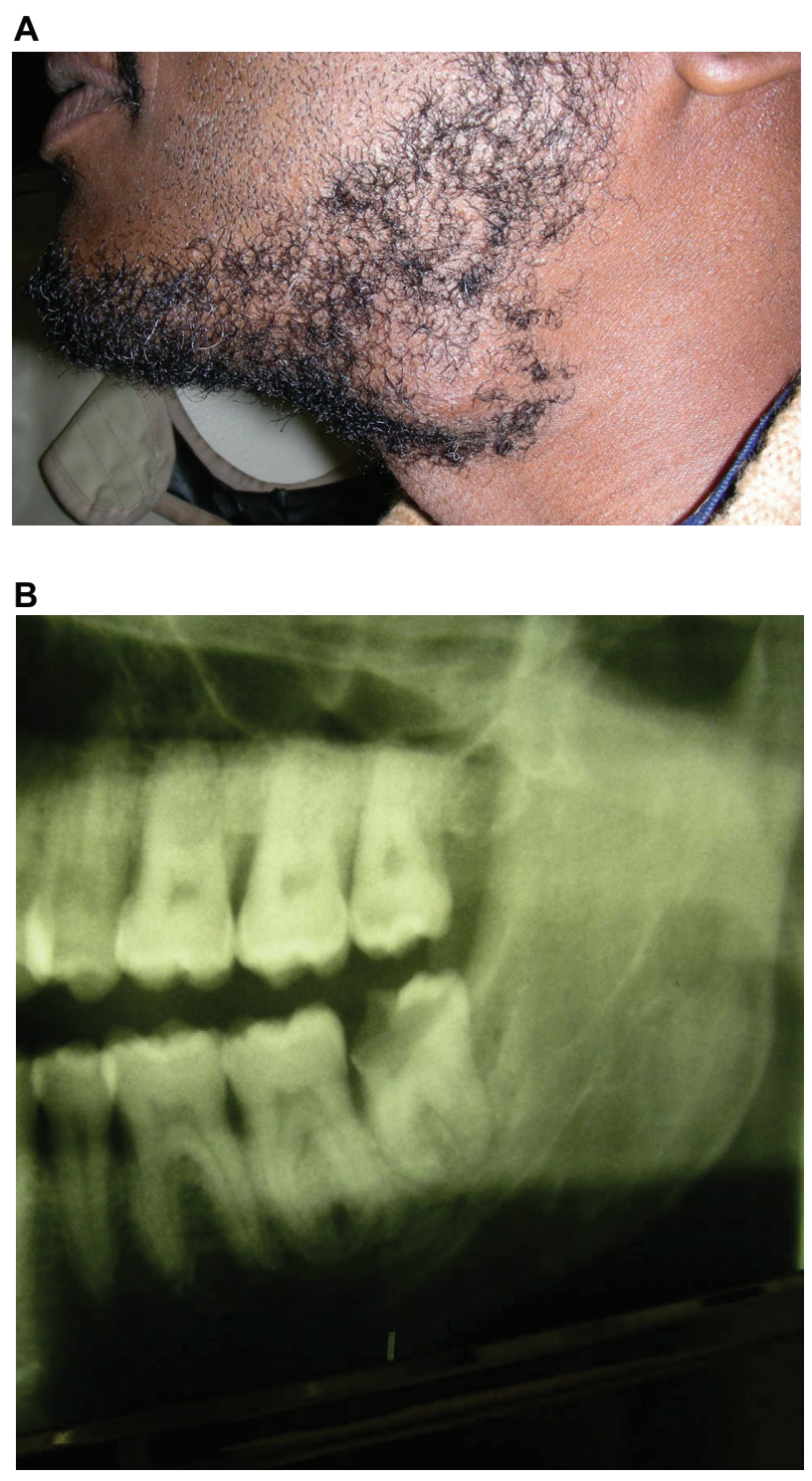

Figure I A) Emerging cutaneous sinus tract on the neck of case no. I patient. B) Panoramic radiograph demonstrating abscessed third molar.

The medical history was negative. The patient reported using a hot or warm washcloth on the left side of his face. Clinical examination revealed an area of firm swelling approximately $1 \mathrm{~cm}$ in diameter slightly superior to the middle of the left ramus of the mandible (Figure 3A). An intra-oral clinical examination revealed the presence of several severely carious teeth and root tips.

A panoramic radiograph was exposed and demonstrated severe carious lesions of the maxillary left canine and first bicuspid and second molar as well as root tips with periapical radiolucencies of the maxillary left first molar and the left mandibular bicuspids (Figure 3B). These teeth were all diagnosed to be non-restorable and the maxillary left first molar and left mandibular bicuspids were noted for a diagnosis of periapical abscess as well. 
A

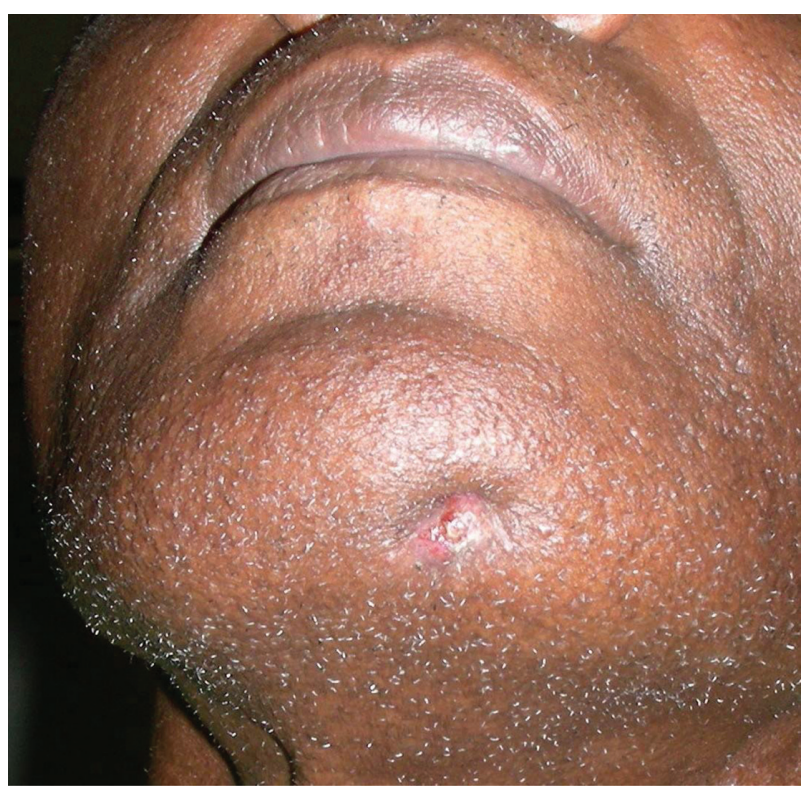

B

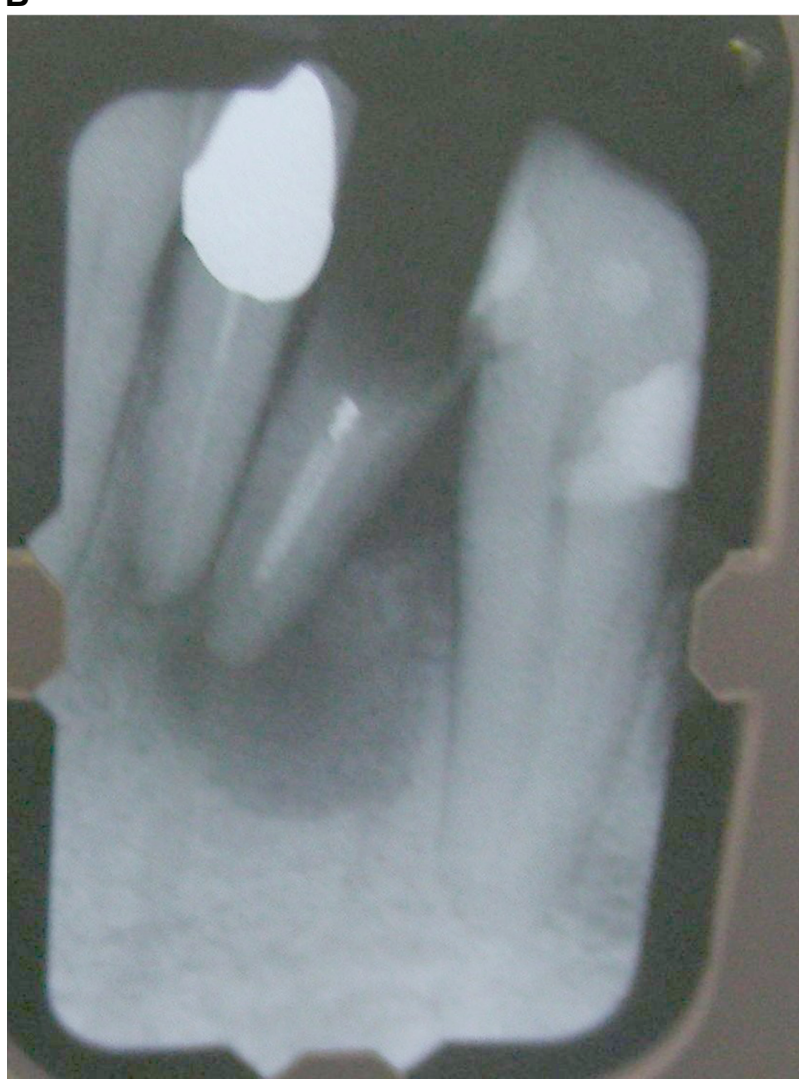

Figure 2 A) Cutaneous sinus tract on the chin of case no. 2 patient. B) Periapical radiograph demonstrating failing root canal therapy of mandibular anterior tooth.

The patient was referred to Oral and Maxillofacial Surgery for the extractions of the noted problematic teeth. The extraction procedures were routine and the patient was lost to follow-up.

\section{A}

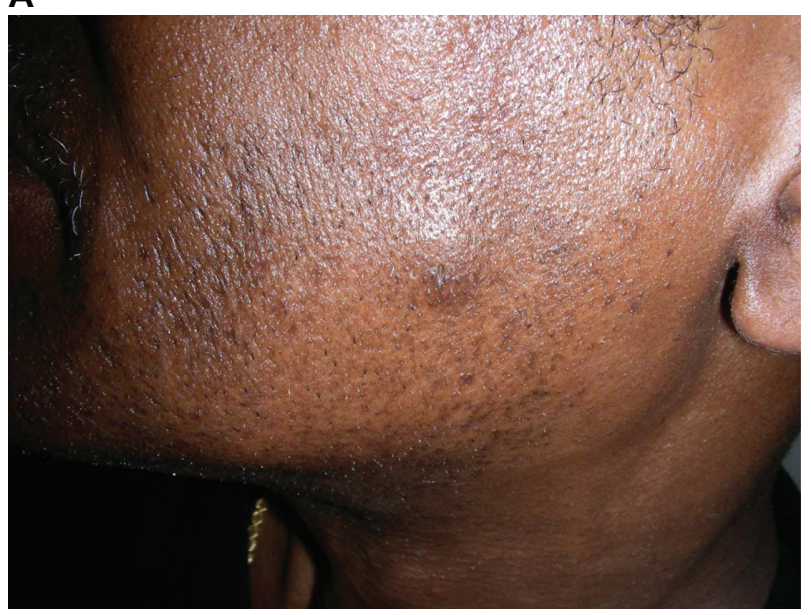

B

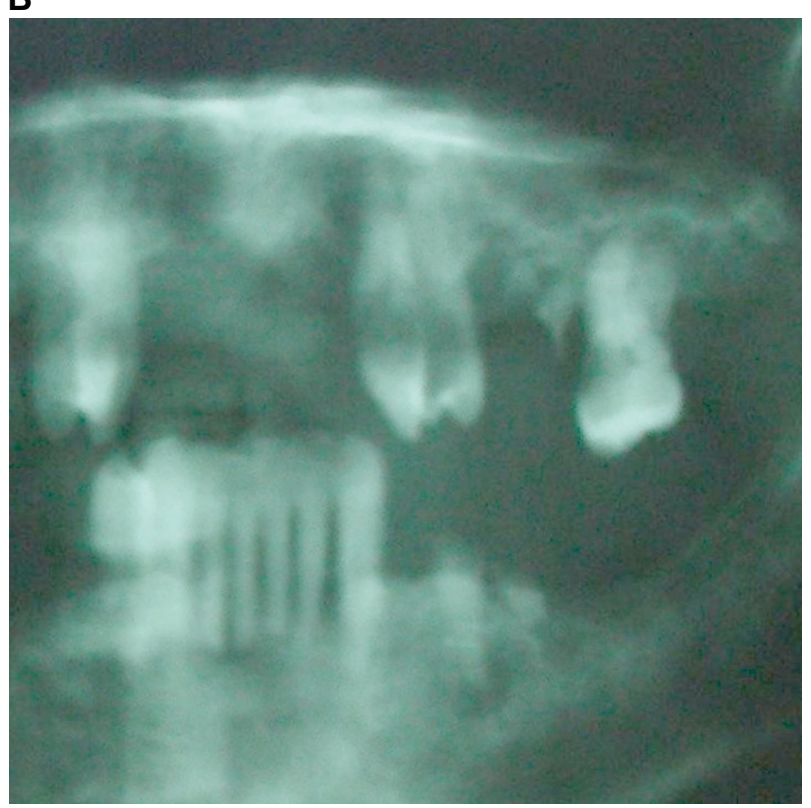

Figure 3 A) Emerging sinus tract on the cheek of case no. 3 patient. B) Portion of panoramic radiograph demonstrating multiple periapical radiolucencies and carious dental lesions.

\section{Discussion}

The most common cause of dental pulp necrosis (periapical abscess) is dental caries. When the tooth is still restorable, endodontic therapy either with or without crown restoration may be a viable means of treatment. However, if the infection is severe or the tooth is not restorable, then extraction is the treatment of choice. ${ }^{4}$

Secondary causes of pulp necrosis include trauma and periodontal infections. The periapical lesion can be either acute or chronic and may remain either localized within the alveolar bone or drain through the periodontal ligament space and outward through the alveolus either intra-orally or extraorally through cutaneous structures. Most periapical abscess 
lesions tend to become acute and, therefore, the patient tends to seek treatment. With chronic infections, however, the local inflammation process may spread slowly through the alveolar bone along the path of least resistance. It may perforate the cortical plate, forming a sub-periosteal abscess and once through the periostium, the infection may then spread into the surrounding soft tissues through facial planes.

If the teeth infections originate superior to the maxillary muscle attachments or inferior to the mandibular muscle attachments, the infection may move through the cutaneous barrier and drain through an extra-oral sinus tract. ${ }^{5}$ Intra-oral fistulation compared to extra-oral sinus tract is the far more common finding. The most common sites of extra-oral sinus tract of odontogenic origin are the jaws and chin with the angle of the jaw being the most common site..$^{1-9}$

Sassone et al performed a microbiologic evaluation of primary endodontic infections in teeth with and without sinus tract. ${ }^{10}$ They discovered the greatest prevalence of such bacteria as Fusobacterium nucleatum sp. vincentii, Porphyromonas gingivalis, Veillonella parvula, Enterococcus faecalis, Campylobacter gracellis, and Neisseria mucosa. The total bacterial counts were similar between lesions with and without sinus tracts, although E faecalis, Streptococcus anginosus, Capnocytophaga sputigena and Capnocytophaga gingivalis had significantly higher counts in those lesions without sinus tracts. Higher levels of $\mathrm{P}$ gingivalis and $F$ nucleatum sp. nucleatum were noted in cases with sinus tract.

Slutzky-Goldberg et al evaluated 1,119 patients referred for endodontic consultation and reported a prevalence of $108(10.4 \%)$ with intra-oral sinus tracts. ${ }^{11}$ They reported that approximately two thirds of sinus tracts originated within the maxilla compared to one third which originated in the mandible. Chronic periapical abscess was the most common cause of sinus tracts of odontogenic origin and was reported in $71 \%$ of the cases. They also found that $82.4 \%$ of these sinus tracts emerged on the buccal aspect of the alveolus and that $12 \%$ of these emerged either on the palatal or lingual aspect of the alveolus.

Johnson et al reported a possible correlation between the application of heat to the face to relieve pain and cutaneous sinus tracts of odontogenic origin. ${ }^{2}$ They surmised that the heat causes vasodilation and increased blood flow to the local area and that such may have contributed to the cutaneous exit of sinus tracts. This contention is supported by the findings of Javid and Barkhordar. ${ }^{9}$ They reported that of 59 patients treated for cutaneous sinus tracts of odontogenic origin, 34 reported using home poultices or hot fomentation to reduce pain and swelling and to draw out the puss. Such therapy may encourage the extraoral release of infection. ${ }^{4}$ One of the three cases reported was noted for utilization of temperature therapy to ameliorate his pain.

Recently, Lee described a surgical technique to aid in the closure of an oral-antral fistula. ${ }^{12}$ His technique involved elevating the maxillary sinus membrane and utilizing the bone-grafting of autogenous ileum bone and a rotated palatal flap in order fill in the defect and preserve the surgical site. Furthermore, Lee utilized the grafted bone for support of dental implants.

Haas et al reported utilizing autogenous monocortical bone blocks harvested from the chin to close large oral-antral fistulous tracts. ${ }^{13}$

Depoi et al reported utilizing platelet rich plasma combined with a flap approach in the corrective surgery of an oral-antral fistula. ${ }^{14}$

Cohen and Eliezri utilized surgical excision within their therapy of a cutaneous sinus tract of dental origin. ${ }^{15}$ However, with regard to the treatment of the three patients reported, surgical correction was not found to be necessary.

In the three cases described, the diagnoses of dental abscesses were relatively straight forward. However, many such cases can present diagnostic dilemmas particularly for physicians not familiar with intra-oral disease entities. These cases may present without obvious dental disease and result in unfocused or inappropriate therapy extending the time of the infection. Patients with cutaneous sinus tracts or emerging cutaneous sinus tracts of dental origin may or may not have experienced dental pain associated with their cutaneous lesion. ${ }^{2,4-6}$ In two of the three cases presented, the origin of the infection was treated previous to the rupture of the skin.

Endodontic therapy is certainly the most appropriate therapy for dental abscesses, however, in all of the three cases presented, the teeth were beyond restorability and endodontic intervention was not appropriate. One of the cases involved a third molar and third molars are seldom treated with endodontic therapy because of the difficulty in performing endodontic therapy because of their posterior position. Also, third molars are not considered appropriate for salvation in most instances because they are not deemed necessary for mastication or speech in most instances.

The gutta percha technique ${ }^{2}$ for locating the origin of a sinus tract was not utilized in any of the cases above. In the second case, the origin was straight forward. In the first case, 
the origin was also straight forward and because the infection had not yet perforated the skin, gutta percha localization was not possible. In the third case, all the involved teeth in the vicinity were non-restorable and designated for extraction and the infection had not yet perforated the skin.

\section{Conclusion}

One case is presented with a cutaneous sinus tract of odontogenic origin and two cases are presented with emerging cutaneous sinus tracts of odontogenic origin. In all three of these cases, the infections were successfully treated with tooth extraction therapy and healing was uneventful.

\section{Disclosure}

The authors report no conflicts of interest in this work.

\section{References}

1. Mittal N, Gupta P. Management of extra oral sinus cases: a clinical dilemma. J Endod. 2004;30:264-267.

2. Johnson BR, Remeikis NA, Van Cura JE. Diagnosis and treatment of cutaneous facial sinus tracts of dental origin. J Am Dent Assoc. 1999; 130:832-836.

3. Cantatore JL, Klein PA, Lieblich LM. Cutaneous dental sinus tract, a common misdiagnosis: a case report and review of the literature. Cutis. 2002;70(5):264-267.
4. Brown RS, Johnson CD, Melissinos EG, Smith BR. A large necrotic defect secondary to a cutaneous sinus tract of odontogenic origin: a case report. Compend Contin Educ Dent. 1995;16:362,264,366,372.

5. Cioffi GA, Terezhalmy CT, Parlette HL. Cutaneous draining sinus tract: an odontogenic etiology. J Am Acad Dermatol. 1986;14:94-100.

6. Hodges TP, Cohen DA, Deck D. Odontogenic sinus tracts. Am Fam Physician. 1989;40:113-116.

7. Tidwell F, Jenkins JD, Ellis CD, Hutson B, Cederberg RA. Cutaneous odontogenic sinus tract to the chin: a case report. Int Endod J. 1997;30: 352-355.

8. Chan CP, Chang SH, Huang CC, Wu SK, Huang SK. Cutaneous sinus tract caused by vertical root fracture. J Endod. 1997;23:593-595.

9. Javid B, Barkhordar RA. Chronic extra-oral fistulae of dental origin. Compend Contin Educ Dent. 1989;108-114.

10. Sassone LM, Fidel R, Faveri M, Fidel S, Figueiredo L, Feres M. Microbiological evaluation of primary endodontic infections in teeth with and without sinus tract. Int Endod J. 2008;41:508-515.

11. Slutzky-Goldberg I, Tsesis I, Slutzky H, Heling I. Odontogenic sinus tracts: a cohort study. Quintessence Int. 2009;40:13-18.

12. Lee B-K. One-stage operation of large oroantral fistula closure, sinus lifting and autogenous bone grafting for dental implant installation. Oral Surg Oral Med Oral Pathol Oral Radiol Endod. 2008;105:707-713.

13. Haas R, Watzak G, Baron M, Tepper G, Mailath G, Watzek G. A preliminary study of monocortical bone grafts for oroantral fistula closure. Oral Surg Oral Med Oral Pathol Oral Radiol Endod. 2003:96:263-266.

14. Depoi R, John V, Paez de Mendoza CY, Gossweiler MK. Development of an oro-antral fistula following sinus elevation surgery: a case report on management using platelet-rich plasma. J Indiana Dent Assoc. 2007-2008;86:10-16.

15. Cohen PR, Eliezri YD. Cutaneous odontogenic sinus simulating a basal cell carcinoma: a case report and literature review. Plast Reconstr Surg. 1990;86:123-127.
Clinical, Cosmetic and Investigational Dentistry

\section{Publish your work in this journal}

Clinical, Cosmetic and Investigational Dentistry is an international, peer-reviewed, open access, online journal focusing on the latest clinical and experimental research in dentistry with specific emphasis on cosmetic interventions. Innovative developments in dental materials, techniques and devices that improve outcomes

\section{Dovepress}

and patient satisfaction and preference will be highlighted. The manuscript management system is completely online and includes a very quick and fair peer-review system, which is all easy to use. Visit http://www.dovepress.com/testimonials.php to read real quotes from published authors. 